\title{
Myelodysplastic syndrome in a kidney transplant recipient after SARS-CoV-2 infection: can SARS-CoV-2 induce myelodysplastic syndrome?
}

\author{
Ivana Juric $^{1}{ }^{1} \cdot$ Lea Katalinic $^{1} \cdot$ Vesna Furic-Cunko ${ }^{1} \cdot$ Nikolina Basic-Jukic $^{1}$
}

Received: 1 September 2021 / Accepted: 12 November 2021 / Published online: 23 November 2021

(c) The Author(s), under exclusive licence to Springer Nature B.V. 2021

Keywords Myelodysplastic syndrome $\cdot$ SARS-CoV-2 $\cdot$ COVID-19 Kidney transplantation

\author{
Abbreviations \\ MDS Myelodysplastic syndrome \\ CMV Cytomegalovirus \\ EBV Epstein-Barr virus \\ HHV-6 Human herpes virus-6 \\ HTLV-1 Human T cell leukemia virus-1 \\ MMF Mycophenolate mofetil
}

Editor,

Numerous microbiological pathogens, mostly viruses, have been involved in the pathogenesis of hematologic malignancies. Different oncogenic viral mechanisms have been described [1]. A myelodysplastic syndrome (MDS) is a group of heterogenous clonal hematopoietic stem cell malignancies characterized by dysplasia and ineffective hematopoiesis. We report a case of MDS after SARS-CoV-2 infection in a kidney transplant recipient.

At the age of 46, a patient received a kidney from a deceased donor with standard immunosuppressive therapy of basiliximab, tacrolimus, MMF and steroids. After delayed graft function in the immediate posttransplant period, good and stable graft function was established. In the later posttransplant course that was otherwise uneventful, MMF was

Ivana Juric

ivanajuric1980@gmail.com

Lea Katalinic

lea_katalinic@hotmail.com

Nikolina Basic-Jukic

nina_basic@net.hr

1 Department of Nephrology, Arterial Hypertension, Dialysis and Transplantation, University Hospital Centre Zagreb, Kispaticeva 12, 10000 Zagreb, Croatia replaced with azathioprine due to intolerance. Nine years after kidney transplantation patient presented with febrility and respiratory symptoms, and was diagnosed with SARSCoV-2 infection with the radiological finding of bilateral pneumonia. He was treated with levofloxacin, steroids, oxygenotherapy and reduced immunosuppressive therapy with good clinical outcome. Regular outpatient follow-up after SARS-CoV-2 infection revealed significant EBV and CMV viremia, previously not detected, while graft function remained stable. Six months after SARS-CoV-2 infection patient presented with severe anemia (hemoglobin $62 \mathrm{~g} / \mathrm{l}$ ) and leucopenia (WBC $1.8 \times 10^{9} / 1$ ) with normal platelet count. Bone marrow puncture revealed abundant hematopoietic tissue with the shift in favor of a red line (1.6:1). Erythrocytopoesis was multiplied, morphologically altered megaloblastoid, binuclear and multinuclear forms with nuclear budding and fragmentation. Granulocytopoiesis was represented predominantly by transient and mature forms while thrombocytopoiesis was moderately abundant and represented by mature and developmental forms of megakaryocytes. Cytomorphological finding suggests MDSs.

Although the precise pathogenesis of MDS remains unclear, two main mechanisms may be involved: the malignant transformation of a pluripotent bone marrow stem cell and immune dysregulation mechanisms, including cytokines production, immune and inflammatory signaling pathways, and immune cells activation [2]. Several studies have discussed the potential role of viral agents in the pathogenesis of the MDS. Viral infection promotes immune system activation, including dysregulated cytokine production creating bone marrow microenvironment and cytokine milieu favoring clonal expansion [3]. Several viruses have been connected with development of MDS in the literature including CMV [4], HTLV-1 [5], parvovirus B19 [6], and HHV-6 [7].

Immune response to SARS-CoV-2 infection is rather diverse and still remains poorly understood. Studies have 
shown heterogeneous CD8, CD4 T cell and B cell response, immune and inflammatory signaling pathways activation and cytokine production [8]. It has been shown that patients with SARS-CoV-2 infection are prone to develop herpesviruses reactivation, including EBV, CMV and HHV-6 [9]. Followup after SARS-CoV-2 infection revealed CMV and EBV viremia in our patient, previously not detected. Based on the existing evidence in the literature, MDS in our patient could be induced by CMV and EBV reactivation promoted by SARS-CoV-2 infection. However, immune response to SARS-CoV-2 infection creates cytokine milieu that could favor clonal expansion and myelodysplastic syndrome development.

In conclusion, we suggest possible causative relation between SARS-CoV-2 and MDS. The SARS-CoV-2 infection could induce MDS development directly or indirectly by creating opportunistic milieu and promoting reactivation of other viruses such as CMV and EBV. Further studies are needed to clarify the possible role of SARS-CoV-2 in the pathogenesis of the MDS.

Acknowledgements All the authors have made an important contribution to the manuscript, are familiar with the data and have read and approved it in its final form. We did not receive financial support for completion of this manuscript, nor are there any direct conflicts of interest to report.

\section{Declarations}

Conflict of interest The authors declare that they have no conflict of interest and they have nothing to disclose.

\section{References}

1. Sadrzadeh H, Abtahi FAT (2012) Infectious pathogens and hematologic malignancy. Discov Med 14(79):421-433
2. Wang C, Yang Y, Gao S, Chen J, Yu J, Zhang H, Li M, Zhan X, Li W (2018) Immune dysregulation in myelodysplastic syndrome: clinical features, pathogenesis and therapeutic strategies. Crit Rev Oncol Hematol 122:123-132. https://doi.org/10.1016/j.critrevonc. 2017.12.013

3. Raza A (1998) Hypothesis: myelodysplastic syndromes may have a viral etiology. Int J Hematol 68(3):245-256. https://doi.org/10. 1016/s0925-5710(98)00051-6

4. Raza A (1998) Initial transforming event in myelodysplastic syndromes may be viral: case for cytomegalovirus. Med Oncol 15(3):165-173. https://doi.org/10.1007/BF02821935

5. Karlic H, Möstl M, Mucke H, Pavlova B, Pfeilstöcker M, Heinz R (1997) Association of human T-cell leukemia virus and myelodysplastic syndrome in a central European population. Cancer Res 57(21):4718-4721

6. Urban C, Lackner H, Müller E, Benesch M, Strenger V, Sovinz P, Schwinger W (2011) Stem cell transplantation in 6 children with parvovirus B19- induced severe aplastic anaemia or myelodysplastic syndrome. Klin Padiatr 223(6):332-334. https://doi.org/ 10.1055/s-0031-1287839

7. Kagialis-Girard S, Durand B, Mialou V, Pagès M-P, Galambrun C, Bertrand Y, Negrier C (2006) Human herpesvirus 6 infection and transient acquired myelodysplasia in children. Pediatr Blood Cancer 47(5):543-548. https://doi.org/10.1002/pbc.20667

8. Mathew D, Giles JR, Baxter AE et al (2020) Deep immune profiling of COVID-19 patients reveals patient heterogeneity and distinct immunotypes with implications for therapeutic interventions. Science. https://doi.org/10.1126/science.abc8511

9. Simonnet A, Engelmann I, Moreau A-S, Garcia B, Six S, El Kalioubie A, Robriquet L, Hober D, Jourdain M (2021) High incidence of Epstein-Barr virus, cytomegalovirus, and human-herpes virus-6 reactivations in critically ill patients with COVID-19. Infect Dis Now 51(3):296-299. https://doi.org/10.1016/j.idnow. 2021.01.005

Publisher's Note Springer Nature remains neutral with regard to jurisdictional claims in published maps and institutional affiliations. 